\title{
On two formulations of an optimal insulation problem
}

\author{
E. Munoz • G. Allaire · M. P. Bendsøe
}

Received: 29 May 2006 / Revised manuscript received: 17 November 2006 / Published online: 20 January 2007

(C) Springer-Verlag 2007

\begin{abstract}
Two formulations for the design of the optimal insulation of a domain are investigated by computational means. The results illustrate the similarities and differences that result from the two approaches. One method is in the format of a topology design problem of distributing insulating material in a domain surrounding a non-design domain that is heated by a given heat source; this problem is treated in both a relaxed format and a penalized material format. The other approach deals with the optimal distribution of a thin layer of insulation on the boundary of the nondesign domain; this problem is more in the realm of shape design, or rather, it is similar to optimal design of support conditions for structures. In both cases, mathematical programming is used, but for the shape design case, it is applied to the non-linear analysis problems that arise when the optimal design is explicitly solved for.
\end{abstract}

Keywords Insulation • Topology optimization • Shape design

E. Munoz $(\bowtie) \cdot$ G. Allaire

Centre de Mathématiques Appliquées, Ecole Polytechnique, 91128 Palaiseau Cedex, France

e-mail: eduardo.munoz-queupumil@polytechnique.org

G. Allaire

e-mail: allaire@cmapx.polytechnique.fr

M. P. Bendsøe

Department of Mathematics,

Technical University of Denmark, 2800 Lyngby, Denmark

e-mail: M.P.Bendsoe@mat.dtu.dk

\section{Introduction}

Much of the fundamental mathematical insight in the area of optimal design for continuum problems was originally obtained by studying the scalar case of conduction (or equivalently, torsion; see, for example,the overviews given in the books by Cherkaev 2000; Allaire 2002). However, only a moderate number of papers in the area cover computational experiments for conduction problems and it is common that the maximization of the conduction is treated, typically measured in such a way that the problem is analogous to the wellknown minimum compliance problem for structures. Even rarer are papers on the design of insulation per se; however, the basics for this case are analogous to the maximization of the torsional stiffness of a shaft. We refer the reader to the papers of Lavrov et al. (1980), Lurie and Cherkaev (1997), Glowinski (1984), Goodman et al. (1986), Pedreira and Vinter (1990), Burns and Cherkaev (1997), Sigmund and Torquato (1997), Cox et al. (1999), Donoso and Sigmund (2004), Li et al. (2004), Zuo et al. (2005), Ha and Cho (2005), and Donoso and Pedregal (2005).

In this paper, we deal with variations of the problem of optimal design of insulation in a common physical setting that is inspired by the work on the "thin insulation" case by Buttazzo (1988b). Inside a given domain $\Omega_{\mathrm{fix}}$, a heat source $f$ is given and this source is restricted to a subdomain $\omega$ of the fixed domain $\Omega_{\text {fix }}$ (Fig. 1). Our goal is to find an optimal use (maximization of heat) of an amount of insulation material to be distributed around the domain $\Omega_{\mathrm{fix}}$; this can be as a layer on the boundary or it can be distributed in a domain surrounding $\Omega_{\mathrm{fix}}$. This is an abstract setting of finding the insulation of a house $\Omega_{\mathrm{fix}}$ with radiators in $\omega$ that 
Fig. 1 Template geometry of the topology design insulation problem with heat source in $\omega$ and design domain $\Omega_{\mathrm{des}}$

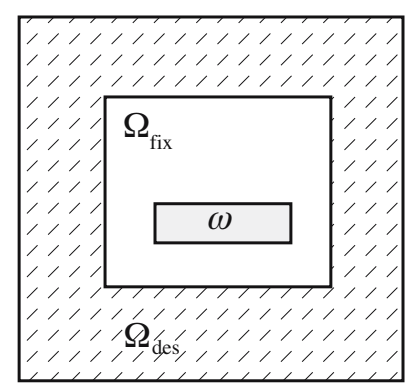

generate heat given by $f$; for simplicity, in this paper, we treat the problem in 2-D.

The paper illustrates several of the issues that have been a central part of the work of Pauli Pedersen to whom this volume of Stuctural and Multidisciplinary Optimization is dedicated: shape design, topology design, the use of optimization algorithms for design and analysis, and work with problem structure.

\section{The topology optimization formulation}

For the topology optimization problem, we imbed the domain $\Omega_{\text {fix }}$ into a larger domain $\Omega$ and use the area $\Omega_{\mathrm{des}}=\Omega \backslash \Omega_{\mathrm{fix}}$ as the design domain.

The analysis problem for the temperature $T$ is the stationary heat transfer equation defined in all of $\Omega$,

$-\nabla(a(x) \nabla T(x))=f$ in $\Omega ; \quad T(x)=0$ on $\partial \Omega$

with zero temperature on the boundary $\partial \Omega$ of $\Omega$. Here, $f$ is the heat source density, which is zero outside of the set $\omega$ (in the examples, we set $f=1$ in $\omega$ ). The parameter $a(x)$ corresponds to the local conductivity of the material. Explicitly, $a(x)$ is the function

$a(x)= \begin{cases}\delta_{\mathrm{con}}, & \text { no insulation at } x \\ \delta_{\mathrm{ins}}, & \text { there is insulation at } x\end{cases}$

where $0<\delta_{\text {ins }} \ll \delta_{\text {con. }}$. Also, we have that $a(x)=\delta_{\text {con }}$ in $\Omega_{\text {fix }}$.

The optimization problem we will treat is one of optimal insulation, that is, we seek an optimal distribution of a given amount $V$ of the insulator with conduction properties $a=\delta_{\text {ins }}$ so as to maximize the heat in the area $\Omega_{\mathrm{fix}}$. The objective for heat is here expressed as (Buttazzo 1988b, note that $f=0$ in $\Omega \backslash \omega$ )

$H=\int_{\omega} f T \mathrm{~d} x=\int_{\Omega_{\mathrm{fix}}} f T \mathrm{~d} x=\int_{\Omega} f T \mathrm{~d} x$

where $T$ is the solution of (1). We note here that the global insulation properties could also be measured in terms of dissipation of heat, e.g., in terms of the fundamental eigenvalue of heat conduction problem as in (1) (for a treatment of this for the case of a thin insulation layer, see Cox et al. 1999).

Using the weak form of the state equation (1), we can here express our goal to find the minimum of the functional

$J(T, a)=\int_{\Omega} a(x)|\nabla T(x)|^{2} \mathrm{~d} x-2 \int_{\Omega} f(x) T(x) \mathrm{d} x$

in both fields. This follows from the property that

$\min _{\substack{\left.T \\ T\right|_{\partial \Omega}=0}} J(T, a)=-H$.

To make the problem nontrivial, we should also impose a volume constraint:

$V_{\text {ins }}(a)=\int_{\Omega_{\text {des }}} 1_{\left\{x: a(x)=\delta_{\text {ins }}\right\}} \mathrm{d} x \leq V$

Elaborating further, the problem we should solve can be written as

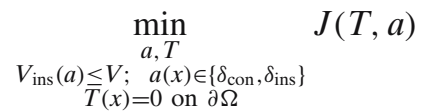

We note that this way of rewriting the optimization problems in this form is also quite standard in studies of minimum or maximum compliance problems (cf., e.g., Cherkaev 2000; Allaire 2002; Bendsøe and Sigmund 2003).

In the following, we shall describe both a solid isotropic material with penalization (SIMP)-type approach and a relaxation approach to deal with this problem. We will here apply a standard nested approach for the computational treatment, meaning that the analysis problem is seen as a function call and the optimization procedure just deals with the optimization over the design field $a$. This means that we solve the problem that can be written as

$\min _{\substack{a, a(x) \in\left\{\delta_{\text {con }}, \delta_{\text {ins }}\right\} \\ V_{\text {ins }}(a) \leq V}}\left[\min _{\substack{T \\ T(x)=0 \text { on } \partial \Omega}} J(T, a)\right]$

\subsection{An approach by penalized interpolation}

For the pure topology design problem, we wish to obtain results that satisfy the discrete valued nature of the constraint (2). However, to avoid the use of integer programming, we adopt here the the SIMP method, which has proven very popular and extremely efficient in structural applications (for an overview, see Bendsøe and Sigmund 2003). In our present setting, it means 
that we introduce a spatially varying density $\rho(x)$ of the insulation material and express the conductivity properties at a point $x$ in the design domain $\Omega_{\text {des }}$ by an expression:

$a_{p}(\rho(x))=(1-\rho(x))^{p}\left(\delta_{\text {con }}-\delta_{\text {ins }}\right)+\delta_{\text {ins }}, \quad 0 \leq \rho \leq 1$

and with the volume constraint written as

$\int_{\Omega_{\mathrm{des}}} \rho(x) \mathrm{d} x \leq V$

In (9), $p$ is a suitably chosen penalty parameter that should infer a penalization of intermediate values of $\rho$. Note that the interpolation (9) satisfies $a_{p}(\rho(x)=0)=$ $\delta_{\text {con }}$ and $a_{p}(\rho(x)=1)=\delta_{\text {ins }}$.

The computational procedure we will apply deals with the nested format (8), which we here write as

$$
\begin{gathered}
\min _{\rho}-H(\rho) \\
\text { s.t. : } \int_{\Omega_{\text {des }}} \rho(x) \mathrm{d} x \leq V \\
0 \leq \rho(x) \leq 1
\end{gathered}
$$

The min-min format of the design problem (8) means that a suitable choice of the power $p \geq 1$ in (9) is different from the typical minimum structural compliance setting. First of all, we note that for $p=1$, (9) is a linear interpolation (like a Voigt upper bound for the effective properties). This means that problem (11), as an optimization problem in $\rho$, for $p=1$ has a concave objective function and a convex constraint set. This follows from (5), which shows that the objective function of (11) is a minimization of linear (i.e., concave) functions. The concavity of the objective function for $p=1$ implies the existence of a globally optimal 0-1 solution for a finite element method (FEM) discretized version of the problem where we, for example, use element-wise constant densities in a uniform mesh and a volume constraint that is an integer times the volume of the base element. This kind of property is also the idea behind the so-called RAMP interpolation proposed in Stolpe and Svanberg (2001) for structural applications.

\subsubsection{Computational method}

The computational solution procedure for (11) follows what one could label the "standard procedure" in the area. The temperature field and the the design field are discretized using FE, here using element-wise constant interpolations for $\rho$ and linear, triangular elements for
$T$ (the elements consist of a triangulation of the square elements used for $\rho$ ). The resulting finite dimensional optimization problem is then solved using sensitivity analysis and MMA (Svanberg 2002). The sensitivity analysis is quite standard (see, e.g., Bendsøe and Sigmund 2003, p.17) and will thus not be repeated here.

Numerical experiments have shown that it is beneficial to use a continuation method for the penalty parameter $p$ (like for SIMP in structural applications). Here a natural continuation method using the interpolation scheme is to begin the optimization procedure with $p$ around 2 to 4 and then decrease $p$ until $p=1$. Finally (and not unexpectedly), one should also, for the present case, apply a filtering technique to avoid mesh-dependent results; in this work, we have used the gradient-filtering techniques originally proposed by Bendsøe and Sigmund (2003, p.35).

\subsubsection{Examples}

Various example results for the topology design setting are shown in Figs. 2, 3, 4, 5, 6, 7, and 8. Unless otherwise stated, all figures using SIMP have been obtained using filtering techniques (cf. the discussion above).

Note that most of the designs place the insulation directly on the boundary of the fixed domain $\Omega_{\mathrm{fix}}$; in essence this means that a simple topology is chosen. However, as shown in Fig. 6, certain combinations of geometry and material parameters can result in a design where the optimal use of insulation is not to place this directly along the boundary of $\Omega_{\mathrm{fix}}$. There a decrease in the conduction properties of the insulation material (by a factor of 7.5 as compared to the left-hand design in Fig. 6) results in a new type of design (to the right) where the insulation material is not "sticking" to the boundary of the domain $\Omega_{\mathrm{fix}}$ - the part of the design domain that is enclosed by the insulation is the hatched region.

We remark here that various types of optimal designs are obtained depending on the data given; this includes the shape of the domains $\Omega_{\text {fix }}$ and $\omega$, as well as the size of the outer design domain $\Omega$. Also, the placement of $\omega$ within $\Omega_{\mathrm{fix}}$ and the placement of $\Omega_{\mathrm{fix}}$ within $\Omega$ plays a role. The later is specifically important as it controls how "close" the boundary of $\Omega_{\mathrm{fix}}$ is to the ambient temperature ( $T=0$; see Fig. 7). One sees that there is a tendency that "classical" solutions are more likely if the insulation and the outer boundary of zero temperature are far apart.

If one wishes to model a situation where the outer temperature $(T=0)$ is applied directly to the outer boundary of the insulation, the format of the optimiza- 


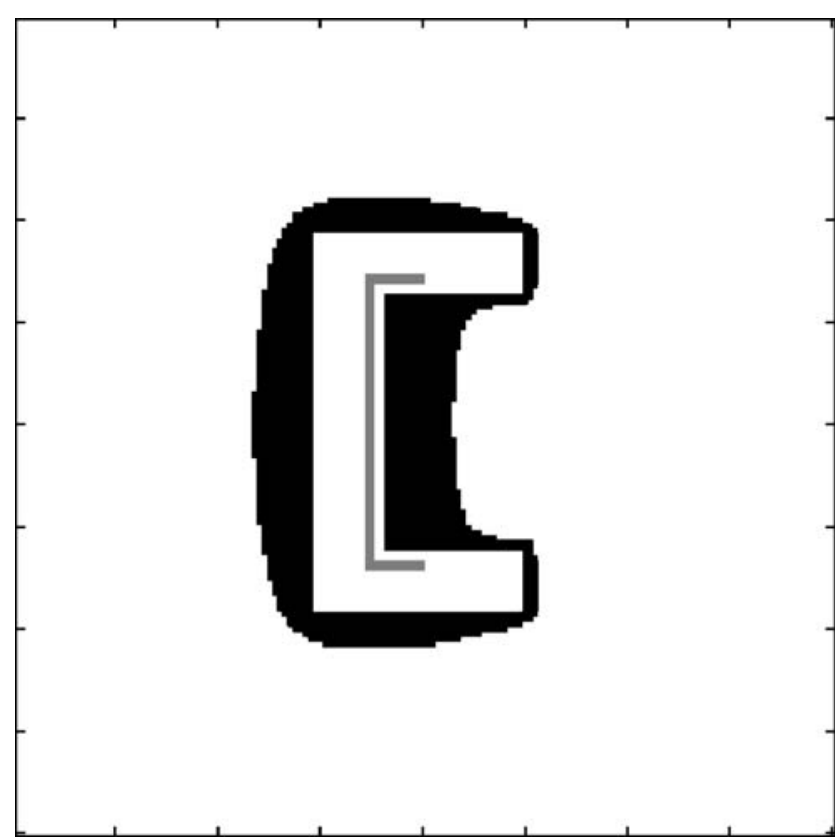

Fig. 2 Optimal design for the topology design insulation problem (using SIMP), with heat source in the gray area. The black area is the insulation that can be placed outside the inner white domain, but only inside the outer square

tion problem should be changed, for example using ideas that have been devised to deal with pressure load problems in structural applications (see, for example, Hammer and Olhoff 2000; Chen and Kikuchi 2001; Sigmund and Clausen 2006).

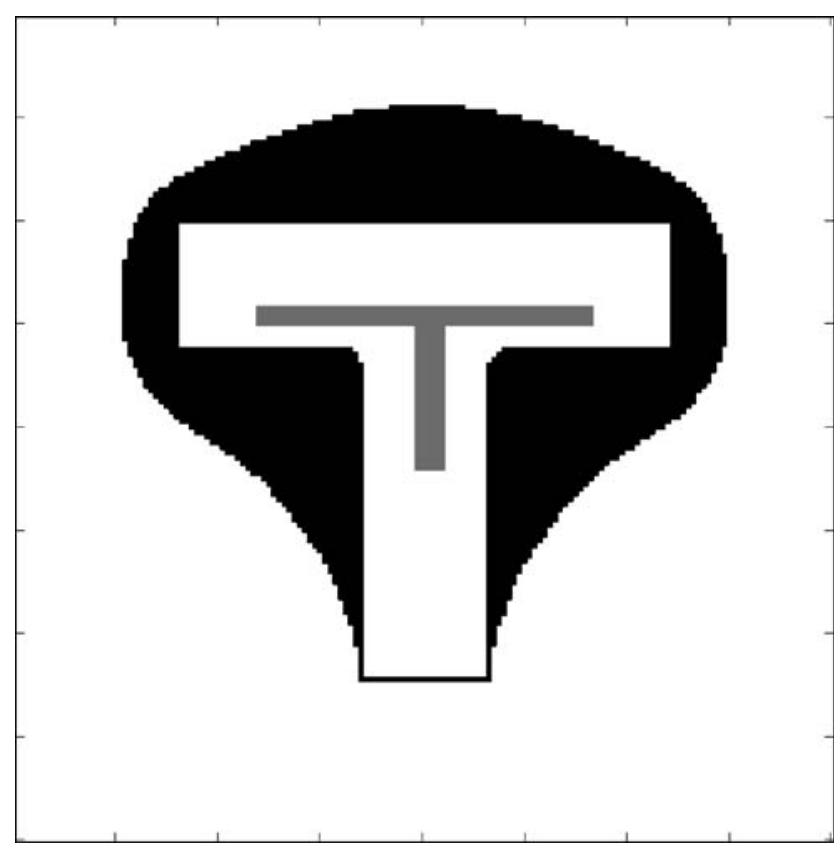

Fig. 3 Optimal design for the topology design insulation problem (using SIMP). Here, insulation is not required along all of the boundary

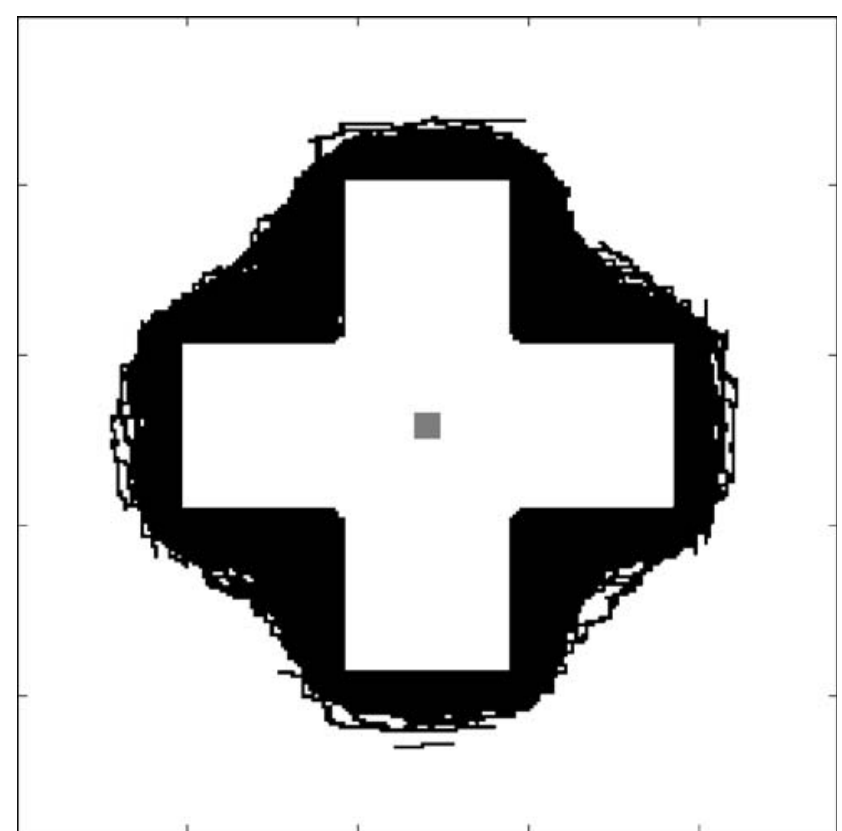

Fig. 4 Optimal design for the topology design insulation problem (using SIMP), without a filtering scheme, using a 240 by 240 mesh

\subsection{Relaxation of the topology optimization formulation: no filtering used}

As is well-known, the integer-valued problem (8) does not generally allow for the existence of solutions in the continuum formulation of the problem. For the problem at hand, it turns out that the relaxation requiredas for structural problems-will involve composites.

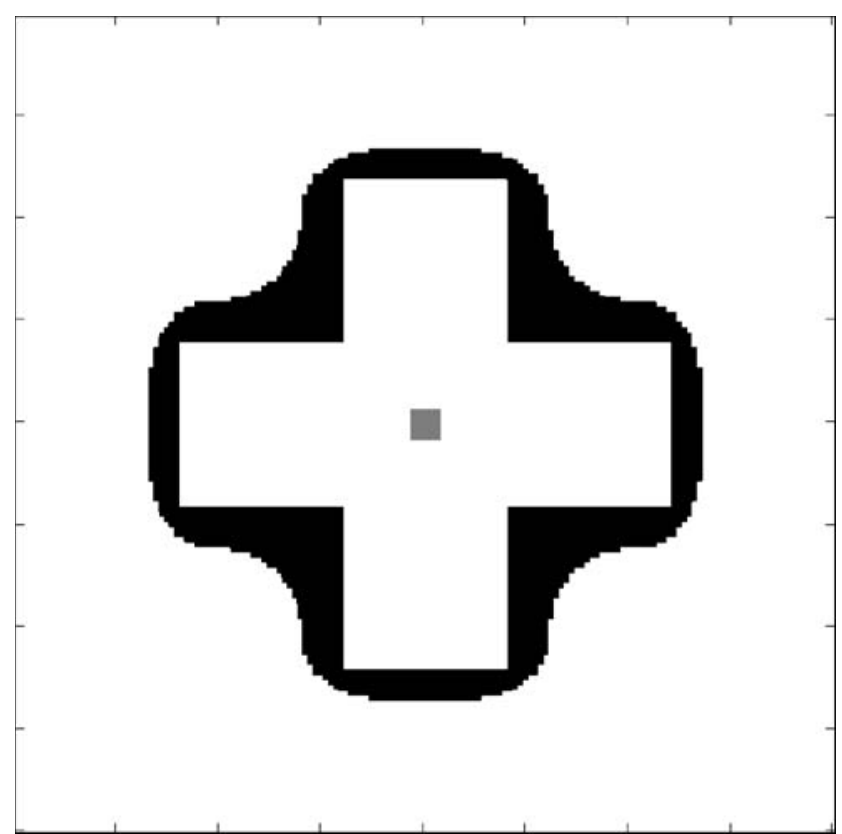

Fig. 5 An example similar to Fig. 3, but now using a filter. Results for a 160 by 160 mesh 

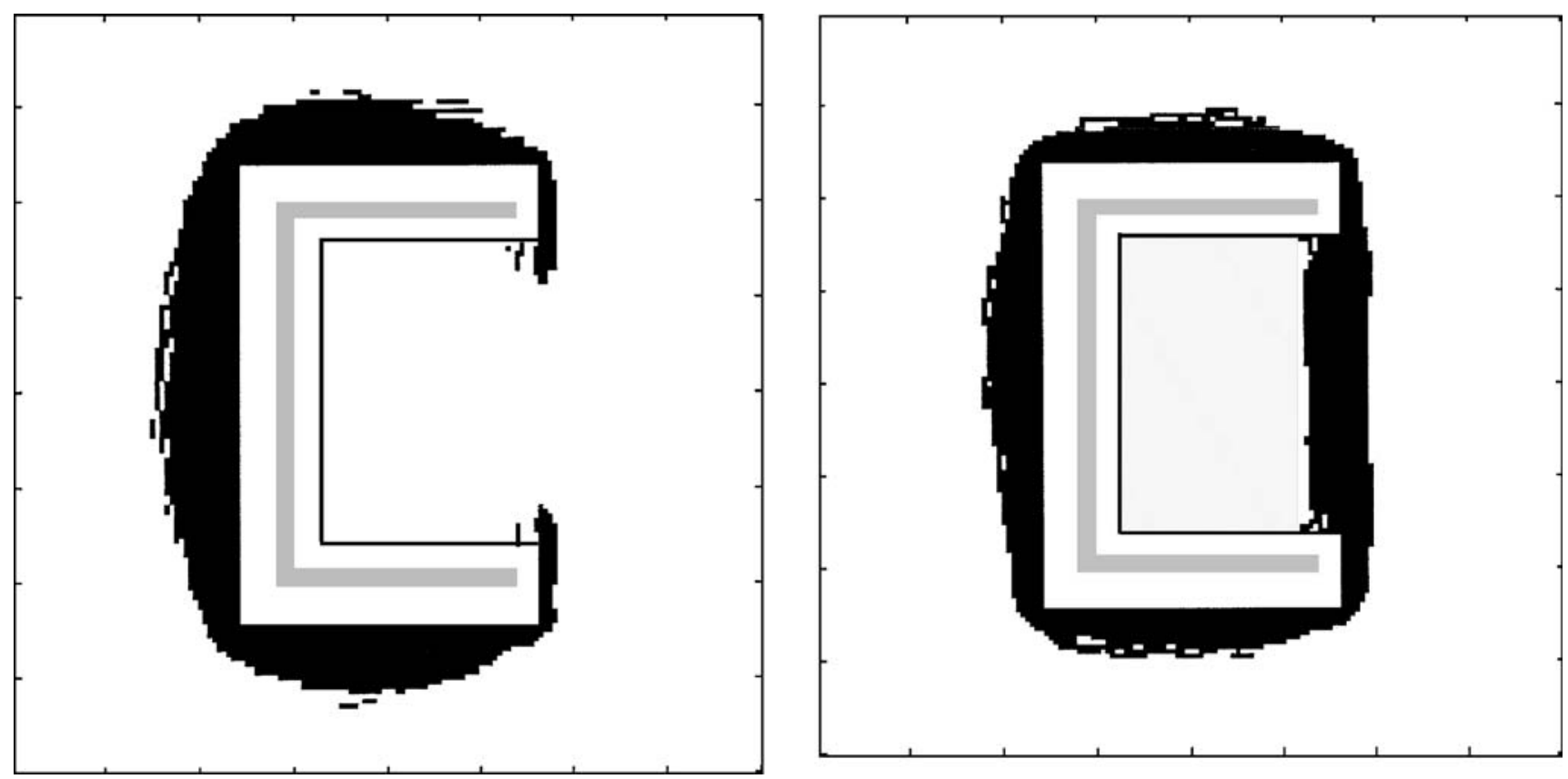

Fig. 6 An illustration that topology may change in some circumstances. Solutions obtained without filtering

However, as we are dealing with a scalar conduction problem and are minimizing heat, the relaxation is the same as the convexification of the problem, which again is equivalent to using the Reuss-like lower bound that interpolates the conduction properties by the harmonic average of the properties (see Lurie and Cherkaev 1997; Murat and Tartar 1997; Cherkaev 2000; Allaire 2002 , and references therein). This means that the problem (11) becomes a convex problem in $\rho$ if we use the interpolation:

$$
\begin{aligned}
a_{\text {relax }}(\rho(x)) & =\left[\rho(x) \frac{1}{\delta_{\text {ins }}}+(1-\rho(x)) \frac{1}{\delta_{\text {con }}}\right]^{-1} \\
& =\frac{\delta_{\text {ins }} \delta_{\text {con }}}{\rho(x) \delta_{\text {con }}+(1-\rho(x)) \delta_{\text {ins }}}
\end{aligned}
$$

The material properties in (12) for intermediate values of $\rho$ can be realized by a layered medium (a socalled rank 1 layering), which has an energy that can be seen as isotropic since the direction of the layering is given by the gradients of the temperature field. Moreover, the energy can be given via material properties defined in (12), which is a Reuss lower bound on the conductivity. Note that this is quite unique for the scalar conduction case at hand (for elasticity, the Reuss lower bound is not attainable by a composite).

\subsubsection{Computational procedure}

If one chooses to use the formulation (11) together with (12), one can follow the same computational procedure as described above for the SIMP interpolation. In this case, however, one does not need a continuation method nor a filtering technique. Moreover, optimal designs do have areas with intermediate values of $\rho$, in contrast to the situation for the SIMP method. An example is shown in Fig. 8. Computations have been performed on a 120 by 120 mesh of squares, and no symmetry conditions have been imposed. Note the appearance of moderate areas with "gray" in the relaxed solution.

We note here that the related computational results contained in Goodman et al. (1986) work with a formulation in the state field only, that is, problem (8) is first solved analytically with respect to the design field $\rho$.

\section{A model with a boundary layer of insulation}

The model we will apply for the shape design of a layer of insulation around a given domain is in the literature called "the thin insulation layer" problem (Fig. 9). In this model, one uses the assumption that the insulation material will be concentrated in a thin layer around the insulated body like a varnish layer, and the design variable is the thickness of the insulation in each point of the body's boundary. The format we will 

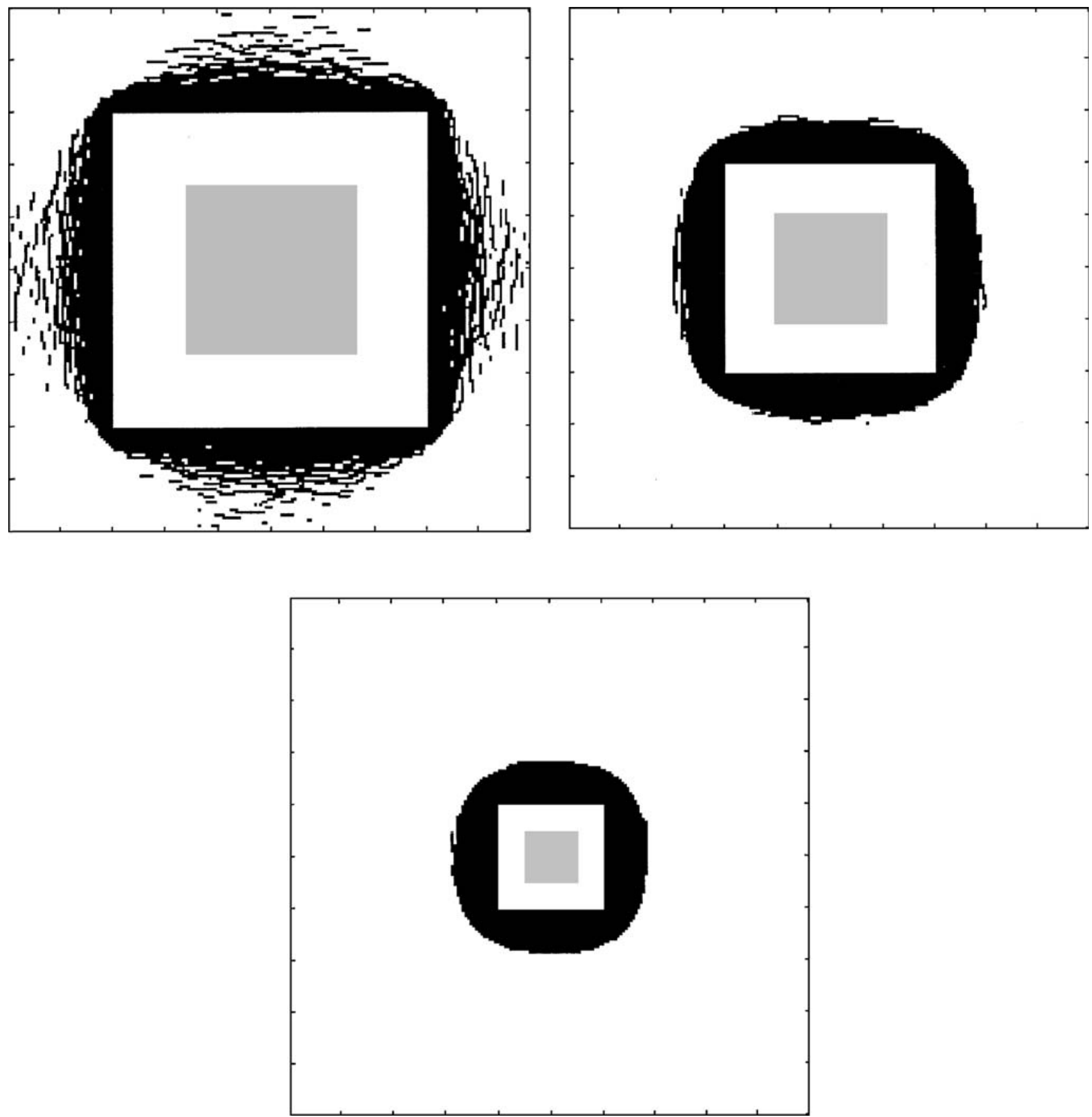

Fig. 7 The influence of choice of outer design domain $\Omega$

use here is the limiting case where the layer is infinitely thin but with infinitely high insulation properties, but with a nontrivial limiting model (neither Dirichlet nor Neuman boundary conditions prevail).

We will first formulate the problem with a finite layer of insulation. Consider now the fixed domain $\Omega_{\mathrm{fix}}$ with a suitable smooth boundary and let $n(x)$ represent the unit outer normal to $\partial \Omega_{\mathrm{fix}}$, for every $x \in \partial \Omega_{\mathrm{fix}}$. We cover the boundary of $\Omega_{\text {fix }}$ by an external thin layer $\Sigma_{\epsilon}$ of in- sulating material of conductivity $0<\delta_{\text {ins }} \ll \delta_{\text {con }}$ as follows. For a thickness function $d \in C^{0}\left(\partial \Omega_{\mathrm{fix}},[0,+\infty]\right)$, let us define the insulation set $\Sigma_{\epsilon}(d)$ as

$\Sigma_{\epsilon}(d)=\left\{x+\operatorname{tn}(x)\right.$, with $\left.\left.x \in \partial \Omega_{\mathrm{fix}}, 0 \leq t<\epsilon d(x)\right)\right\}$

where $\epsilon>0$ is small enough. The complete model will then be defined on the set $\Omega_{\epsilon}:=\Omega_{\mathrm{fix}} \cup \Sigma_{\epsilon}(d)$ and the 
conductivity properties in this domain will be given as (for notational simplicity, we set here $\delta_{\text {con }}=1$, and write $\delta_{\text {ins }}=\delta$ ):

$a_{\epsilon, \delta}(x):= \begin{cases}1, & x \in \Omega_{\mathrm{fix}} \\ \delta, & x \in \Sigma_{\epsilon}\end{cases}$

The analysis problem for the temperature $T$ is as earlier (1) given on the set $\Omega_{\epsilon}$, with heat source $f$. In this
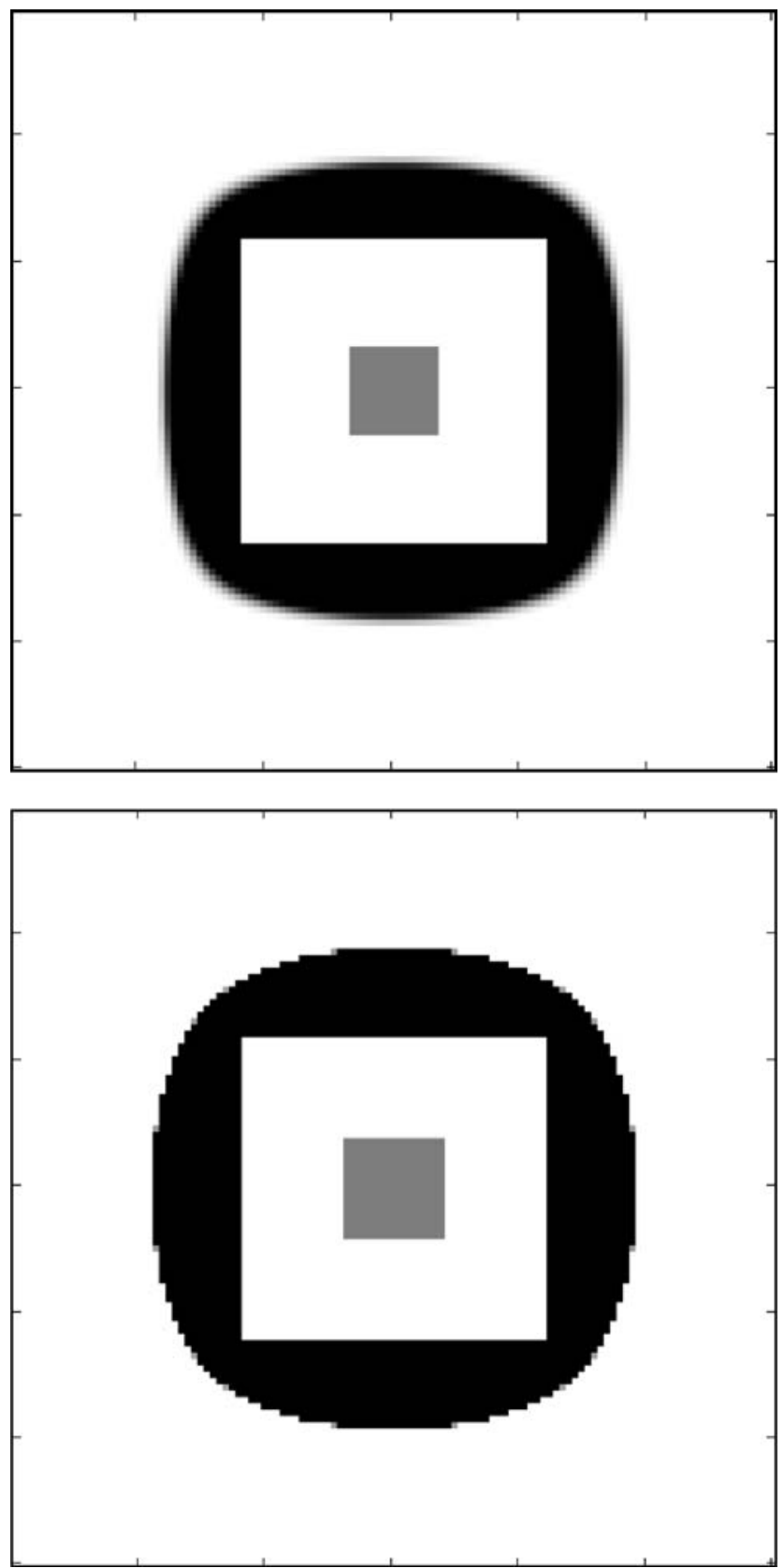

Fig. 8 Optimal insulation of a square with an internal square heat source. Top A solution to the relaxed design problem. Below The corresponding case solved with SIMP
Fig. 9 Template geometry of the thin insulation problem, with heat source in $\omega$ and thin design domain $\Sigma_{\epsilon}$

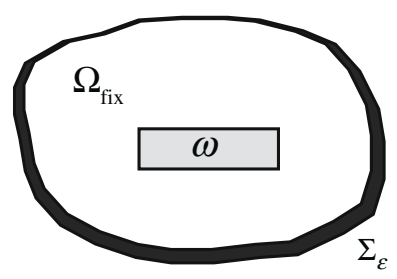

new setting, the variational formulation of the state equation can be written as

$$
\min _{\substack{T \\ T \mid \partial \Omega_{\epsilon}=0}} \int_{\Omega_{\mathrm{fix}}}|\nabla T|^{2} \mathrm{~d} x+\delta \int_{\Sigma_{\epsilon}}|\nabla T|^{2} \mathrm{~d} x-2 \int_{\Omega_{\mathrm{fix}}} f T \mathrm{~d} x
$$

It is known that when considering the asymptotic behavior of problem $(15)$ when $(\epsilon, \delta) \rightarrow(0,0)$, we have to consider three distinct cases, depending on the convergence rates of $\epsilon$ and $\delta$. First, if $\epsilon \ll \delta$, then the limiting case is where the insulation could be neglected, and the limit is the classical Dirichlet problem defined on $\Omega_{\text {fix }}$. In the second case where $\epsilon \gg \delta$, the limit case is such that there is no conduction in $\Sigma_{\epsilon}$ and the limiting case will be that of the classical Neuman problem on $\Omega_{\text {fix }}$.

The third possibility is of interest for optimal design. In this case, $\epsilon \approx \delta$, that is, $\epsilon=k \delta$, where $k$ is a constant, and the limit case represents the situation where the width of the insulating layer and the conductivity of the insulating material go "equally fast" to zero $(\delta=$ $\epsilon / k$, and $\epsilon \rightarrow 0$ ). The correct meaning of "limit case" should be given in terms of $\Gamma$-convergence (see, for example, Buttazzo 1988a,b; Buttazzo and Kohn 1987; Morini 2002; Esposito and Riey 2003) and becomes

$\min _{T} E(T, d)$

with

$E(T, d)=\int_{\Omega_{\mathrm{fix}}}|\nabla T|^{2} \mathrm{~d} x-2 \int_{\Omega_{\mathrm{fix}}} f T \mathrm{~d} x+\int_{\partial \Omega_{\mathrm{fix}}} \frac{T^{2}}{k d} \mathrm{~d} x$

In partial differential equation (PDE) form, this problem has the form:

$-\Delta T=f$ in $\Omega_{\mathrm{fix}}, \quad k d \frac{\partial T}{\partial n}+T=0$ on $\partial \Omega_{\mathrm{fix}}$

which has a boundary condition that mixes Dirichlet and Neuman data. 


\subsection{The optimization setting}

In order to find the "best" insulation around $\Omega_{\text {fix }}$, our optimization problem for the model (16) will be formulated analogously to (8). The design fields will be positive thickness functions $d$ defined on the boundary of $\Omega_{\text {fix }}$, which satisfy a volume constraint. Thus, the design set is

$\Gamma_{w}=\left\{d \in L^{1}\left(\partial \Omega_{\mathrm{fix}}\right): d \geq 0, \int_{\partial \Omega_{\mathrm{fix}}} d \mathrm{~d} x \leq w\right\}$

and the optimization problem of maximizing heat in the domain $\Omega_{\mathrm{fix}}$ becomes the convex problem: ${ }^{1}$

$\min E(T, d)$.

$\underset{d \in \Gamma_{w}}{d, T}$

The convexity of (20) means that one could probably, with advantage, solve the problem computationally using an optimization procedure that works simultaneously with both the design and the temperature field. However, here we take another approach and, instead, solve analytically for the design field in (20). That is, rewriting the problem as

$\min _{T} \min _{d \in \Gamma_{w}} E(T, d)$,

we solve the inner problem directly. That is, we use that for every field $T$, in which there exists a solution $d_{T} \in$ $\Gamma_{w}$ (which is unique if $T$ is nonzero) of the minimum problem

$\min \left\{\int_{\partial \Omega_{\mathrm{fix}}} \frac{T^{2}}{k d} \mathrm{~d} x: d \in \Gamma_{w}\right\}$

and this solution is given as

$d_{T}=w \frac{|T|}{\int_{\partial \Omega_{\mathrm{fix}}}|T| \mathrm{d} x}$.

Inserting (23) in (21), we obtain the following equivalent problem in the state field $T$ only:

$\min _{T}\left\{\int_{\Omega_{\mathrm{fix}}}|\nabla T|^{2} \mathrm{~d} x-2 \int_{\Omega_{\mathrm{fix}}} f T \mathrm{~d} x+\frac{1}{k w}\left(\int_{\partial \Omega_{\mathrm{ffix}}}|T| \mathrm{d} x\right)^{2}\right\}$

This is the problem that we then will solve by computational means. It is a pure analysis problem of finding a minimizer of a potential energy, albeit of a rather unusual form. This is due to the last term of the energy in (24), which has the form of a penalization of the temperature on the boundary of the domain; the

\footnotetext{
${ }^{1}$ This follows from the strict convexity of the real function $(x, y) \rightarrow x^{2} / y, y>0$, in two variables.
}

lesser insulation available, the more the temperature is penalized. However, note that the penalization is not of standard type as it couples the behaviour at all points on the boundary.

Note that, having obtained the optimal field $T$, we can subsequently compute the optimal design from (23).

\subsection{Computational setup}

One should first note that one cannot solve (24) in a classical way by the finite element method. To see this, one should note that the PDE format corresponding to (24) is of the form

$$
\begin{aligned}
-\Delta T & =f & & \text { in } \Omega_{\mathrm{fix}} \\
0 & \in k w \frac{\partial T}{\partial n}+H(T) \int_{\partial \Omega_{\mathrm{fix}}}|T| \mathrm{d} x & & \text { on } \partial \Omega_{\mathrm{fix}}
\end{aligned}
$$

where $H(t)$ is the multi valued mapping

$H(t)= \begin{cases}\operatorname{sign}(t) & \text { if } t \neq 0 \\ {[-1,1]} & \text { if } t=0\end{cases}$

The problem (25) is a non-local PDE as the boundary condition in each point involves the integral of $|T|$ over the whole of the boundary. This is not easy to solve by standard methods (i.e., by solving linear equations). However, we remark that if the source term $f$ is nonnegative, then - by the maximum principle - the temperature, for any design, is positive. Therefore, there is no need (in such a case) for the absolute value in (24). Consequently, the underlying PDE (25) becomes linear (albeit it is still nonlocal).

Here, we have thus chosen to use FE for the discretization, but to solve the resulting finite dimensional optimization problem by an optimization algorithm.

\subsubsection{Smoothing the functional}

To solve the problem (24), we have choosen a finite element space of linear elements on triangular elements so that one can express the functional (24) in terms of the expansion coefficients of the FEM approximation. Moreover, in order to work with a smooth objective function, we approximate the absolute value term of (24) by the expression

$|T| \approx \sqrt{T^{2}+\gamma^{2}}$

with $\gamma \ll 1$.

The resulting nonlinear but convex optimization problem in the FE expansion coefficients was then solved by using the SNOPT optimization algorithm (Gill et al. 2005). SNOPT is a general purpose system 


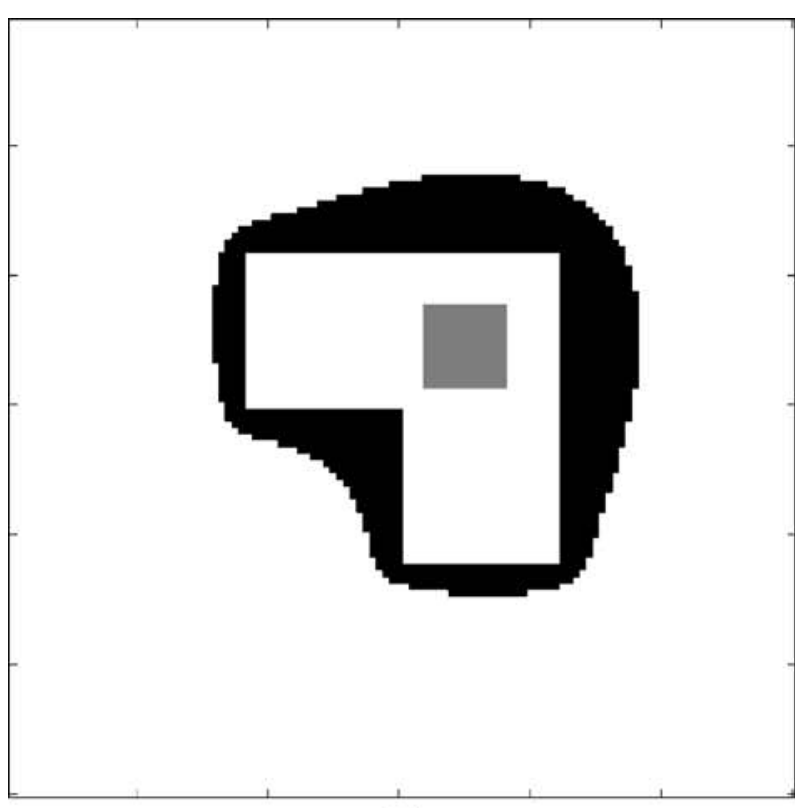

a
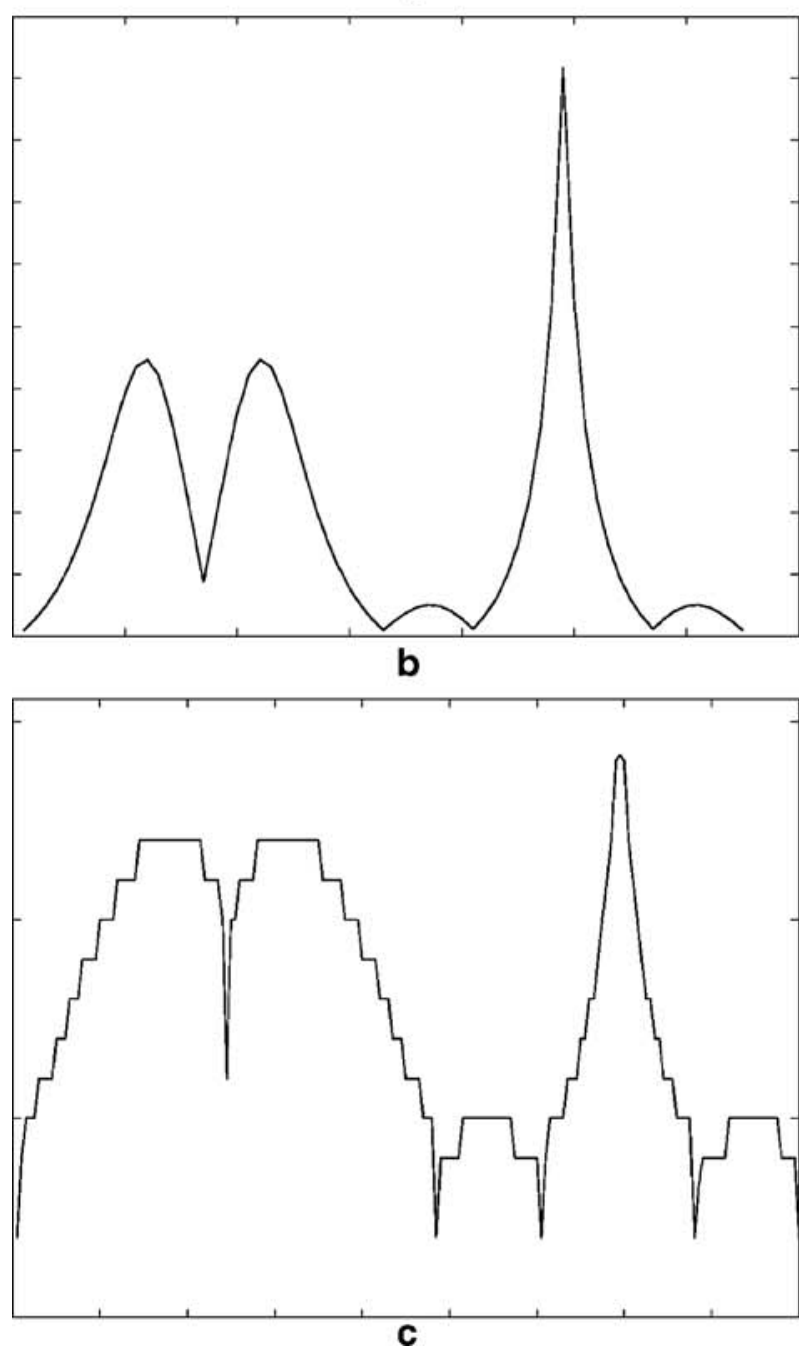

Fig. 10 Comparing SIMP and the thin insulation case for solving linear or nonlinear optimization problems involving many variables (and constraints) by a sequen tial quadratic programming algorithm. After finding the field $T$ for the optimal design, one can evaluate its values on the boundary to find the optimal thickness distribution of insulation [using (23)].

\subsection{Examples}

In Figs. 10, 11, and 12, we show a range of examples that illustrate the thin insulation case. The results are illustrated by showing a graph of the optimal insulation thickness, as it is distributed around the boundary. Comparisons with the topology design case (the SIMP variant) are also shown. There are here two ways to compare the results; one is by computing the thickness of the insulation layer in the topology example. An alternative is to compare the values of the temperature field along the boundary of the domain using the expression

$d=\frac{|T|}{\int_{\partial \Omega_{\mathrm{fix}}}|T| \mathrm{d} x}$

which is the optimal layer thickness in the thin insulation case.

Figure 10 shows the geometry of an insulation problem for a "two-winged house" and the topology optimization solution together with the distribution of the optimal thin layer over the boundary; thus the graph in Fig. 10b shows the thickness $d_{T}$ as a function of the position along the boundary, moving clockwise and

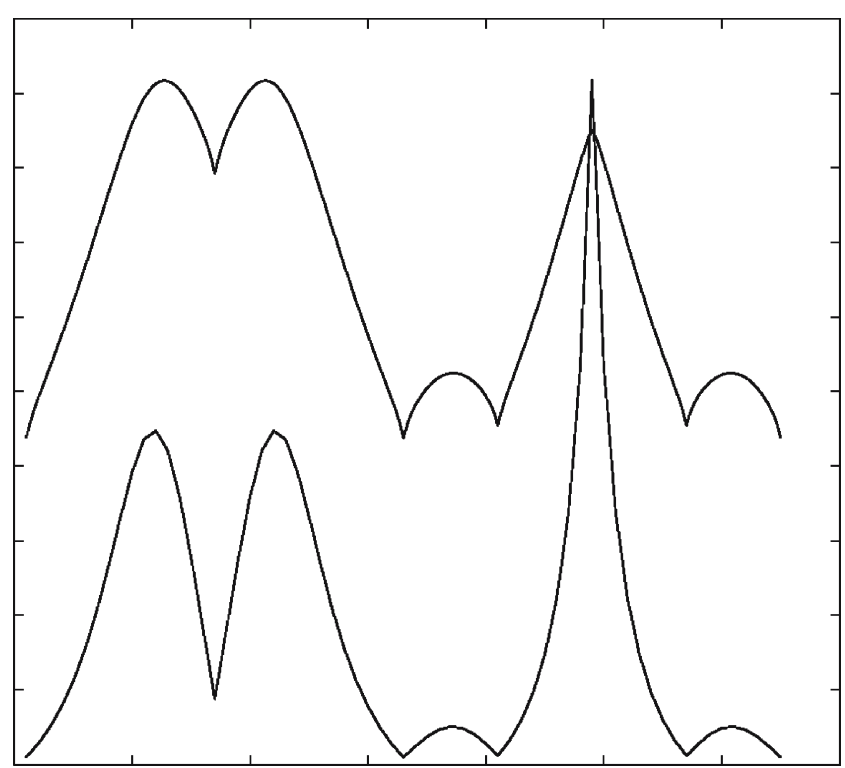

Fig. 11 A direct comparison of the temperature along the boundary of $\Omega_{\mathrm{fix}}$ for the designs obtained in Fig. 10 


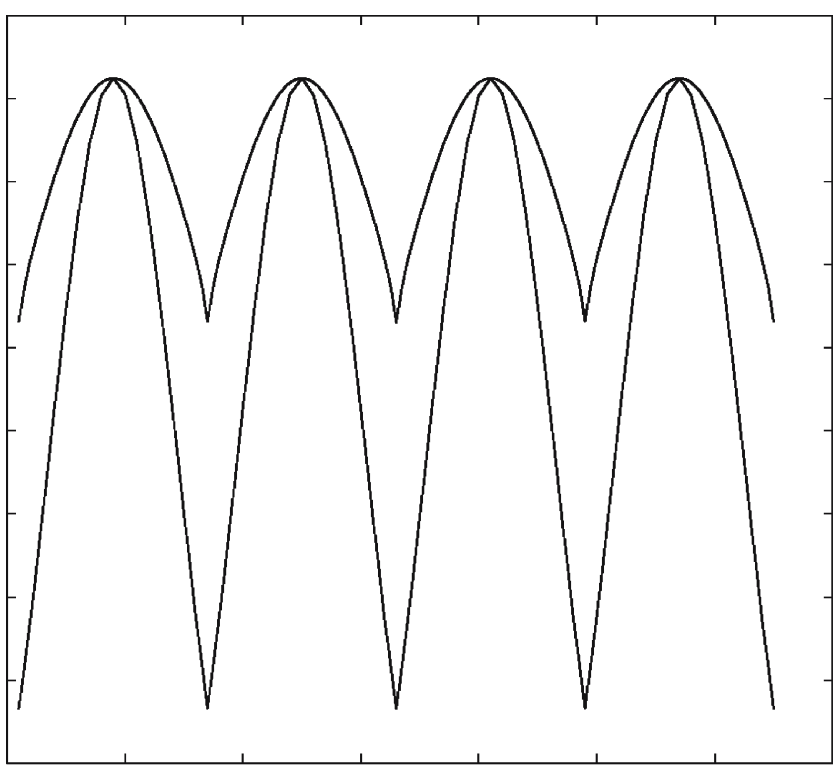

a

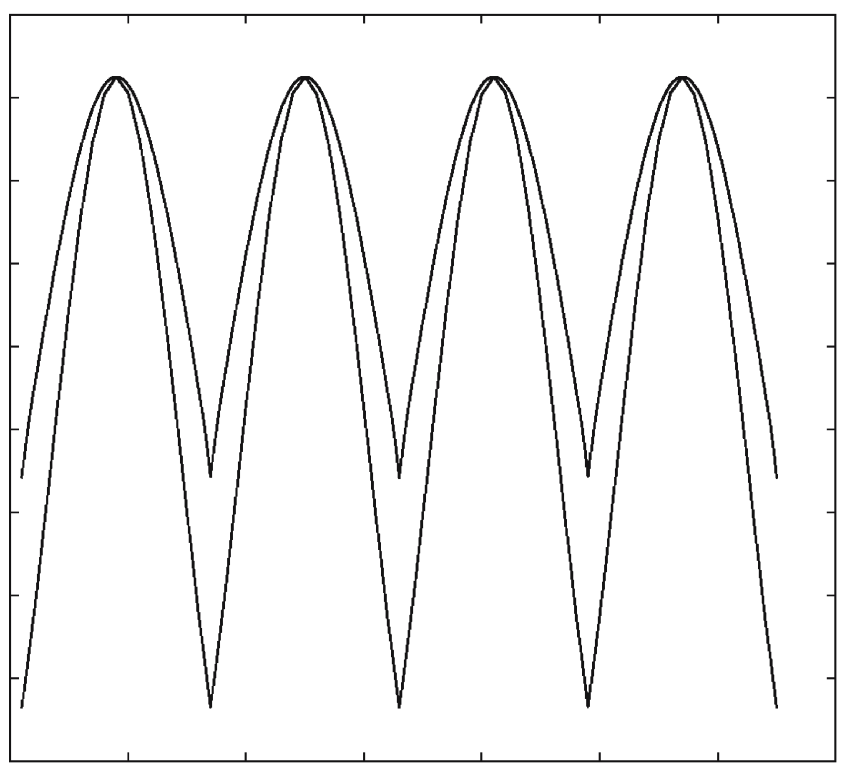

C

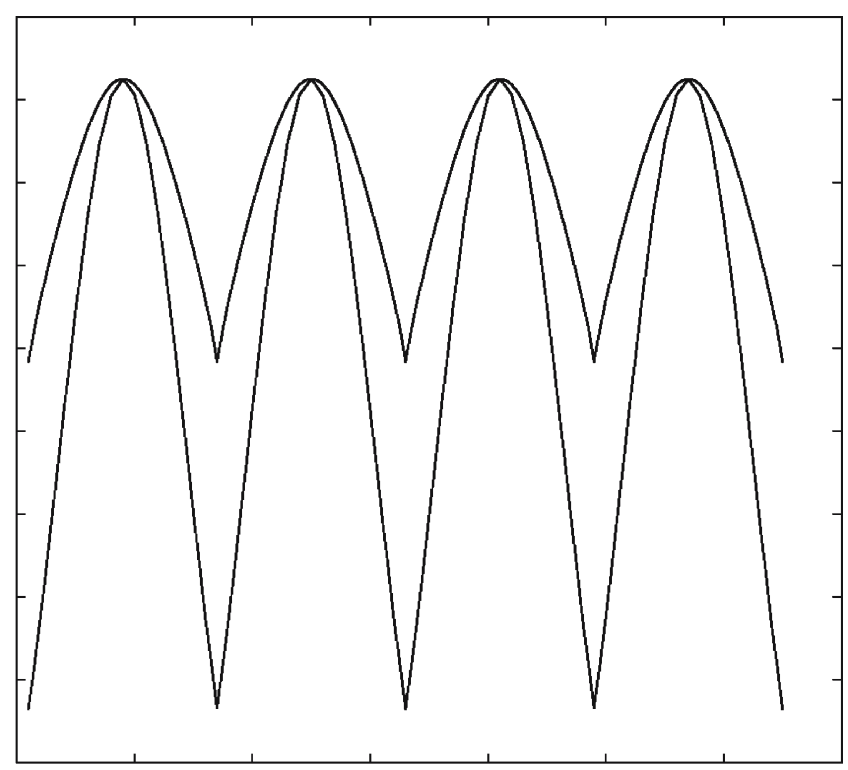

b

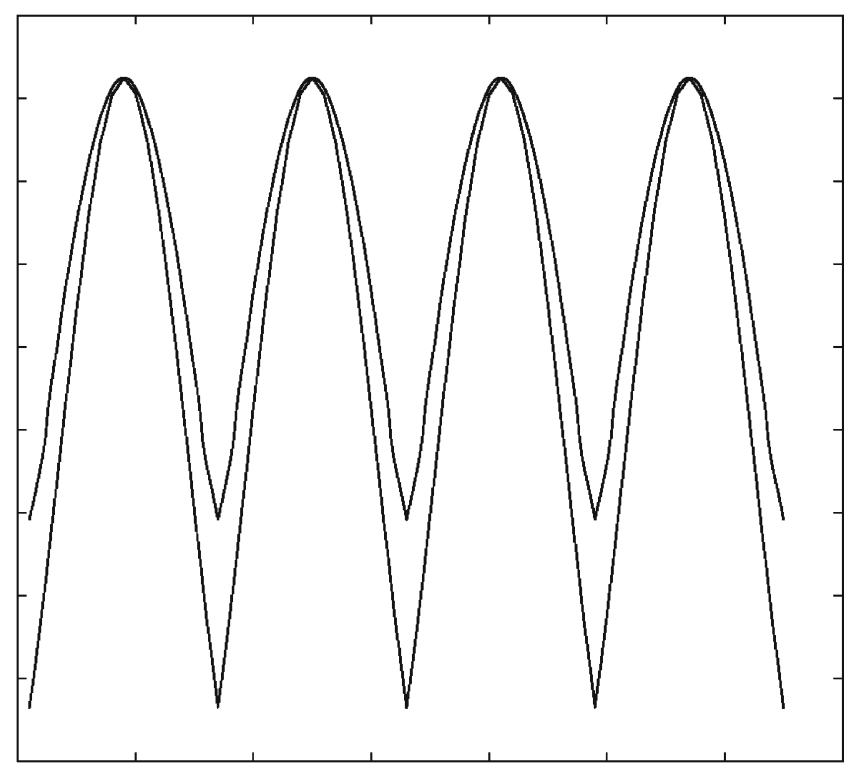

d

Fig. 12 An illustration of the convergence of the topology optimized designs to the thin insulation case

starting to the left at the left-most upper hand corner of the domain $\Omega_{\mathrm{fix}}$. Finally, for comparison, Fig. 10c shows the thickness of the insulation layer in the topology optimized case, measured along the normal to the boundary of $\Omega_{\mathrm{fix}}$ (and illustrated as in Fig. 10(b)). When using the relationship (28) one obtains the two graphs of Fig. 11, where the lower curve is for the thin insulation case, the upper for the topology design case. Note the similar physics of the two cases.

Using (28) also allows us to directly compare the behavior of the topology design problem when one in this formulation decreases the volume of insulation material, while at the same time, the conductivity of the insulating material is decreased. As seen in Fig. 12, this can effectively demonstrate the convergence, as predicted by theory. The graphs show the temperature around the outer boundary of $\Omega_{\mathrm{fix}}$ for the geometry shown in Fig. 8 for the thin insulation case (lower curve in all four plots) and for the topology design, where the volume is lowered and the insulation properties made higher. Note that finer meshes are required when reducing the volume, in order to have proper resolution. 
The four plots thus correspond to an FEM mesh resolution of (a) $60 \times 60$, (b) $80 \times 80$, (c) $100 \times 100$, and (d) $120 \times 120$, for the full analysis domain $\Omega$.

\section{Conclusions}

The methods of topology and shape design can effectively be applied to a broad range of physics. In this paper, we have dealt with the problem of optimal insulation, using a whole range of the theoretical and computational techniques and results of the field. The problem is somewhat unique in its mathematical structure and it allows for illustrating many features of the area in a rather compact form.

Acknowledgements This work was performed for a large part during a visit for 4 months by E. Munoz to the Technical University of Denmark over the summer of 2005 and was supported by the Villum Kann Rasmussen Foundation. This support is gratefully acknowledged. The implementation of the method of moving asymptotes used here was provided by Prof. Krister Svanberg from the Department of Mathematics at KTH in Stockholm. We thank Prof. Svanberg for allowing us to use his program.

\section{References}

Allaire G (2002) Shape optimization by the homogenization method. Springer, Berlin Heidelberg New York

Bendsøe MP, Sigmund O (2003) Topology optimization-theory, methods and applications. Springer, Berlin Heidelberg New York

Burns T, Cherkaev AV (1997) Optimal distribution of multimaterial composites for torsional beams. Struct Optim 13(1):1423

Buttazzo G (1988a) An optimization problem for thin insulating layers around a conducting medium. In: Boundary control and boundary variations. Lecture notes in computer science, vol 100. Springer, Berlin Heidelberg New York, pp 91-95

Buttazzo G (1988b) Thin insulating layer: the optimization point of view. In: Ball JM (ed) Material instabilities in continuum mechanics and related problems. Oxford University Press, Oxford, pp 11-19

Buttazzo G, Kohn R (1987) Reinforcement by a thin layer with oscillating thickness. Appl Math Optim 16(3):247-261

Chen BC, Kikuchi N (2001) Topology optimization with designdependent loads. Finite Elem Anal Des 37:57-70

Cherkaev AV (2000) Variational methods for structural optimization. Springer, Berlin Heidelberg New York

Cox SJ, Kawohl B, Uhlig PX (1999) On the optimal insulation of conductors. J Optim Theory Appl 100(2):1573-2878

Donoso A, Pedregal P (2005) Optimal design of 2D conducting graded materials by minimizing quadratic functionals in the field. Struct Multidiscipl Optim 30:360-367

Donoso A, Sigmund O (2004) Topology optimization of multiple physics problems modelled by Poisson's equation. Latin American Journal of Solids and Structures 1(2):169-189
Esposito P, Riey G (2003) Asymptotic behaviour of a thin insulation problem. J Convex Anal 2:379-388

Gill PE, Murray W, Saunders MA (2005) SNOPT: an SQP algorithm for large-scale constrained optimization. SIAM Rev 47(1):99-131

Glowinski R (1984) Numerical simulation for some applied problems originating from continuum mechanics. In: Trends in applications of pure mathematics to mechanics, Symp., Palaiseau/France 1983, Lect Notes Phys, vol 195. Springer, Berlin Heidelberg New York, pp 96-145

Goodman J, Kohn RV, Reyna L (1986) Numerical study of a relaxed variational problem from optimal design. Comput Methods Appl Mech Eng 57:107-127

Ha SH, Cho S (2005) Topological shape optimization of heat conduction problems using level set approach. Numer Heat Transf B Fundam 48(1):67-88

Hammer VB, Olhoff N (2000) Topology optimization of continuum structures subjected to pressure loading. Struct Multidiscipl Optim 19:85-92

Lavrov N, Lurie K, Cherkaev A (1980) Non-uniform rod of extremal torsional stiffness. Mech Solids 15(5):74-80

Li Q, Steven G, Xie Y, Querin O (2004) Evolutionary topology optimization for temperature reduction of heat conducting fields. Int J Heat Mass Transfer 47(23):5071-5083

Lurie KA, Cherkaev AV (1997) Effective characteristics of composite materials and the optimal design of structural elements. In: Cherkaev AV, Kohn RV (eds) Topics in the mathematical modelling of composite materials. Progress in nonlinear differential equations and their applications, vol 31. Birkhäuser, Boston, MA, pp 175-258 [English translation of a paper from Uspekhi Mekhaniki (Advances in Mechanics; 1986) 9(2):3-81]

Morini M (2002) An optimal control problem in thin insulating layers theory. Tech. Rep. 02-CNA-024, Center for Nonlinear Analysis, Department of Mathematical Sciences, Carnegie Mellon University, Pittsburgh, PA, http:// www.math.cmu.edu/cna/pub2002.html

Murat F, Tartar L (1997) Calculus of variations and homogenization. In: Cherkaev AV, Kohn RV (eds) Topics in the mathematical modeling of composite materials. Progress in nonlinear differential equations and their applications, vol 31. Birkhäuser, Boston, pp 139-173 [English translation of Calcul des Variations et Homogénéisation, Les Méthodes de l'Homogénéisation Théorie et Applications en Physique, Coll. Dir. Etudes et Recherches EDF, 57(1985), Eyrolles, Paris, pp 319-369]

Pedreira C, Vinter R (1990) On numerical results for shape optimization. In: Amouroux M, El Jai A (eds) Control of distributed parameter systems 1989. Selected papers from the 5th IFAC Symposium, Pergamon, New York, pp 473-477

Sigmund O, Clausen P (2006) Topology optimization using a mixed formulation: an alternative way to solve pressure load problems. Comput Methods Appl Mech Eng, In press, available on-line, doi:10.1016/j.cma.2006.09.021

Sigmund O, Torquato S (1997) Design of materials with extreme thermal expansion using a three-phase topology optimization method. J Mech Phys Solids 45(6):1037-1067

Stolpe M, Svanberg K (2001) On the trajectories of penalization methods for topology optimization. Struct Multidiscipl Optim 21:128-139

Svanberg K (2002) A class of globally convergent optimization methods based on conservative convex separable approximations. SIAM J Optim 12(2):555-573

Zuo K, Chen L, Zhang Y, Wang S (2005) Structural optimal design of heat conductive body with topology optimization method. Chin J Mech Eng 41(4):13-16 